\title{
Characterization of the Interaction between the Cohesin Subunits Rad21 and SA1/2
}

\author{
Nenggang Zhang ${ }^{19}$, Yunyun Jiang ${ }^{1,29}$, Qilong Mao', Borries Demeler ${ }^{3}$, Yizhi Jane Tao²*, \\ Debananda Pati ${ }^{1,2 *}$
}

1 Texas Children' Cancer Center, Department of Pediatric Hematology/Oncology, Baylor College of Medicine, Houston, Texas, United States of America, 2 Department of Biochemistry and Cell Biology, Rice University, Houston, Texas, United States of America, 3 Department of Biochemistry, University of Texas Health Science Center, San Antonio, Texas, United States of America

\begin{abstract}
The cohesin complex is responsible for the fidelity of chromosomal segregation during mitosis. It consists of four core subunits, namely Rad21/Mcd1/Scc1, Smc1, Smc3, and one of the yeast Scc3 orthologs SA1 or SA2. Sister chromatid cohesion is generated during DNA replication and maintained until the onset of anaphase. Among the many proposed models of the cohesin complex, the 'core' cohesin subunits Smc1, Smc3, and Rad21 are almost universally displayed as tripartite ring. However, other than its supportive role in the cohesin ring, little is known about the fourth core subunit SA1/SA2. To gain deeper insight into the function of SA1/SA2 in the cohesin complex, we have mapped the interactive regions of SA2 and Rad21 in vitro and ex vivo. Whereas SA2 interacts with Rad21 through a broad region (301-750 aa), Rad21 binds to SA proteins through two SA-binding motifs on Rad21, namely N-terminal (NT) and middle part (MP) SA-binding motif, located at 60-81 aa of the N-terminus and 383-392 aa of the MP of Rad21, respectively. The MP SA-binding motif is a 10 amino acid, $\alpha$-helical motif. Deletion of these 10 amino acids or mutation of three conserved amino acids $\left(\mathrm{L}^{385}, \mathrm{~F}^{389}\right.$, and $\left.\mathrm{T}^{390}\right)$ in this $\alpha$ helical motif significantly hinders Rad 21 from physically interacting with SA1/2. Besides the MP SA-binding motif, the NT SAbinding motif is also important for SA1/2 interaction. Although mutations on both SA-binding motifs disrupt Rad21-SA1/2 interaction, they had no apparent effect on the Smc1-Smc3-Rad21 interaction. However, the Rad21-Rad21 dimerization was reduced by the mutations, indicating potential involvement of the two SA-binding motifs in the formation of the two-ring handcuff for chromosomal cohesion. Furthermore, mutant Rad21 proteins failed to significantly rescue precocious chromosome separation caused by depletion of endogenous Rad21 in mitotic cells, further indicating the physiological significance of the two SA-binding motifs of Rad21.
\end{abstract}

Citation: Zhang N, Jiang Y, Mao Q, Demeler B, Tao YJ, et al. (2013) Characterization of the Interaction between the Cohesin Subunits Rad21 and SA1/2. PLoS ONE 8(7): e69458. doi:10.1371/journal.pone.0069458

Editor: Daniela Cimini, Virginia Tech, United States of America

Received May 6, 2011; Accepted June 11, 2013; Published July 12, 2013

Copyright: (c) 2013 Zhang et al. This is an open-access article distributed under the terms of the Creative Commons Attribution License, which permits unrestricted use, distribution, and reproduction in any medium, provided the original author and source are credited.

Funding: This study was supported by award 1RO1 CA109330 from the National Cancer Institute to D. Pati, the 2008 Virginia and L.E. Simmons Family Foundation Collaborative research award (https://www.collaborativeresearchfund.org/) to D. Pati and Y. J. Tao, and the Welch Foundation award R4079 to Y. J. Tao. The Ultrascan development is supported by National Institutes of Health grant RO1 RR022200 to B. Demeler. The funders had no role in study design, data collection and analysis, decision to publish, or preparation of the manuscript.

Competing Interests: The authors have declared that no competing interests exist.

*E-mail: pati@bcm.edu (DP); ytao@rice.edu (YJT)

9 These authors contributed equally to this work.

\section{Introduction}

DNA replication in the S-phase produces two identical copies of the chromosomal DNA, called sister chromatids. The sister chromatids are held together until the metaphase-to-anaphase transition, when they are segregated to opposite poles. Holding the sister chromatids together, or cohesion, is accomplished by a multi-protein, ring-like complex called cohesin. Cohesin is composed of four core subunits, Smc1, Smc3, $\operatorname{Rad} 21$, and the stromal antigen protein SA1 or SA2 [1-3]. Smc1 and Smc3 are rod-shaped proteins containing ATP-binding cassette (ABC)-like ATPase motifs. They fold into intramolecular anti-parallel coiled coils and jointly form a V-shaped Smc1/Smc3 heterodimer through their hinge domains [4-6]. The $\mathrm{N}$ - and C-termini of each SMC molecule form an ATPase head domain at the distal end of the two coiled-coils arms [5]. Rad21 binds to the ATPase heads of Smc3 and Smc1 via its N- and C- terminus, respectively, resulting in the formation of a contiguous tripartite ring [5]. Besides the four core subunits, several other proteins, Pds5A, Pds5B, Sororin, and Wapl, also associate with the cohesin complex [7-12].

In higher eukaryotes, cohesins are removed from chromosomes in two phases, the prophase and the anaphase. In the prophase, most of cohesins are removed from the chromosome arms by Pds5-Wapl complex after the cohesins are phosphorylated by kinase Plk1 [26-29]. Recent studies indicate that the cohesinassociated protein Sororin coordinates Plkl-mediated chromosomal arm separation [12,13]. Calcium-inducible cleavage of Rad21 by Calpain-1 also promotes chromosomal arm separation [14]. At the onset of the anaphase, the centromeric and residual arm cohesins are removed by a Separase-mediated cleavage of $\operatorname{Rad} 21$ at residues $\mathrm{R}^{172}$ and $\mathrm{R}^{450}[15,16]$, culminating in separation of the sisters [32-34].

Based on the molecular associations of cohesin subunits, we have provided evidence for a handcuff model of the cohesin complex, which consists of two rings $[17,18]$. Each ring has one set of Rad21, Smc1, and Smc3 molecules. The handcuff is established 
after two Rad21 molecules are orientated in anti-parallel fashion and enforced by either SA1 or SA2 and potentially other cohesinassociated factors. Sister chromatids are held together by one of the two rings. Failure in the formation and maintenance of sister chromatid cohesion results in premature chromatid segregation, which is thought to be a major pathway to aneuploidy, a characteristic feature of most if not all human cancers [19]. A recent study indicated that the SA2 gene is mutated in a number of human tumors, including glioblastoma and melanoma, and targeted inactivation of SA2 leads to sister chromatid cohesion defects and aneuploidy, suggesting a direct role of SA2 in the development of human cancer [20].

In human, SA2 is more abundant than SA1 [2], and the two do not bind to the same cohesin complex [17]. SA2 is present predominantly in the cohesin complex at the chromosomal arm and centromeric regions, whereas SAl has been described to be responsible for the telomeric cohesion [21]. SA1 and SA2 share $\sim 70 \%$ sequence identity with the homology, mostly located in the region of 69-1075 aa of SA1. However, the role of SA1/SA2 proteins, other than their association with the tripartite ring and their essential function in maintaining chromosomal cohesion, has not been fully investigated. It remains unclear if $\mathrm{SA} 1 / 2$ is required for the assembly and maintenance of the cohesin ring, or if the physical association of SAl/2 and Rad21 takes place through direct binding or indirect interaction requiring additional cohesinassociated components. Although the region of 362-403 aa of Rad21 has been reported to bind to SA1 [22], the fine mapping of the $\operatorname{Rad} 21-\mathrm{SAl} / 2$ interaction domains and the functional mechanism of $\mathrm{SAl} / 2$ in chromosomal cohesion have not been described.

Using an array of biochemical and cell biological methods, we mapped the amino acid interactive regions of the SA2 and Rad21 and found that SAl/2 physically interacts with $\operatorname{Rad} 21$ through a region from 301 to 750 aa. We also identified two SA-binding motifs of Rad21. Deletion or mutation of these two Rad21 domains (61-80 aa and 383-392 aa) disrupts the interaction of $\operatorname{Rad} 21$ and $\mathrm{SA} 1 / 2$, as well as some degree of cohesin dimerization that might be mediated through $\mathrm{SAl} / 2$. Importantly, the mutant Rad21 cannot efficiently rescue the premature sister chromatid separation caused by the knockdown of endogenous $\operatorname{Rad} 21$, indicating the structural and physiological importance of these two SA-binding motifs of human $\operatorname{Rad} 21$.

\section{Results}

\section{The region 301-750 aa of the SA2 protein interacts with Rad21}

To map the region(s) of SA2 that physically interacts with $\operatorname{Rad} 21$, we utilized the eukaryotic insect cell expression system to obtain recombinant human SA2 and Rad21 proteins. Baculoviruses overexpressing 6xHis-tagged SA2 and Flag-tagged Rad21 were generated. During the purification of SA2 from Sf21 cells, we noted that the full-length SA2 underwent partial degradation, resulting in a product of $\sim 120 \mathrm{kDa}$ in size, the N-terminal 6xHis tag of which remained intact according to immunoblot (Figure S1A). An in-gel digestion by trypsin and chymotrypsin followed by mass spectrometry (MS) of the digested products indicated that the $120 \mathrm{kDa}$ protein was comprised of the first 1122 residues of SA2 (Figure S1B). Based on these results, we conclude that the degradation of SA2 during purification occurred after amino acid residue $\mathrm{T}^{1122}$.

A secondary structure prediction analysis using the program PredictProtein [23] showed an unstructured random coil region flanking $\mathrm{T}^{1122}$, which likely renders the expressed protein susceptible to proteolytic cleavage. Considering that the unstructured region may function as a domain linker, we generated two new baculoviruses, one expressing the first 1051 amino acid residues of SA2, with the entire unstructured coil region removed, and the other expressing the remainder of SA2 from residues 1052 to 1231 , both containing a $6 \mathrm{xHis}$ tag at the N-terminus. Fulllength Rad21 (1-631 aa), Flag-tagged at the N-terminus, was then expressed in Sf21 insect cells along with either SA2 (1-1051 aa) or SA2 (1052-1231 aa). Co-purification assays were performed using either Ni-NTA affinity beads to pull down the 6xHis-SA2 or the anti-Flag M2 affinity resin (agarose beads conjugated with Flag monoclonal antibody) to pull down Flag-Rad21. The samples were analyzed by Western blot (Figure 1A). Insect cells infected with $\operatorname{Rad} 21$ and the $6 \mathrm{xHis}$-tagged PA protein of influenza A virus were used as a negative control for the co-purification assays. The reciprocal Ni-NTA and Flag co-purification results indicated that SA2 (1-1051 aa) but not the C-terminal SA2 (1052-1231 aa) can co-purify with $\operatorname{Rad} 21$, suggesting that the $\mathrm{N}$-terminal 1051 aa are sufficient and the C-terminal 180 aa of SA2 are dispensable for the Rad21-SA2 interaction in vitro (Figure 1A).

To further narrow down the region of SA2 responsible for the Rad21-SA2 interaction, we generated baculoviruses overexpressing progressive SA2 deletion mutants with 150 amino acids increments/decrements from either the $\mathrm{N}$ - or the C-terminus of SA2 (1-1051 aa) (Figure 1B). As before, Flag-tagged Rad21 was expressed in Sf21 cells along with each of the 6xHis-tagged SA2 deletion mutants. Co-purifications were performed with Ni-NTA and Flag beads and analyzed by Western blot as described above. Co-purification results showed that the N-terminal (1-300 aa) and the C-terminal (751-1051 aa) regions of SA2 are not critical for interaction with $\operatorname{Rad} 21$ (Figure 1C). With the exception of SA2 (1$903 \mathrm{aa}$ ), other SA2 fragments containing the amino acid region of 301-750 interacted with Rad21 (Figure 1C, lanes 17-19, 21 \& 3233, 35-37). The interaction between $\operatorname{Rad} 21$ and SA2 (1-903 aa) was very weak (Figure 1C, lanes $20 \& 34$ ) and apparently was not due to less protein in the input sample (Figure 1G, lane 6). The interaction of $\operatorname{Rad} 21$ and SA2 (1-903 aa) might be reduced by a disturbed structure or protein misfolding. Indeed, a protein domain prediction using the program GlobPlot [24] showed that SA2 (850-940 aa) might form a globular domain, which supports our reasoning. Based on the information above, we conclude that the region of SA2 interacting with $\operatorname{Rad} 21$ is located within 301750 aa region.

\section{SA2 interacts directly with the Rad21 central region in Sf21 insect cell system}

Previous studies have shown that yeast Scc3 directly interacts with Sccl via the C-terminal Separase cleavage fragment [5]. In humans, Rad21 is cleaved by Separase at the onset of anaphase at $\mathrm{R}^{172}$ and $\mathrm{R}^{450}[16]$. To determine the region of the human Rad21 that interacts with SA2, Rad21 deletion mutants were designed with reference to the two Separase cleavage sites and the corresponding baculoviruses were generated (Figure 2A). SA2 (1-1051 aa) was expressed in Sf21 cells along with Rad21 or its deletion mutants, and co-purification of the complex was performed to examine the interaction between SA2 and the Rad21 deletion mutants (Figure 2B). Insect cells infected with 6xHis-tagged SA2 (1-1051 aa) and Flag-tagged PA protein were used as a negative control for the co-purification assays. The results showed that SA2 (1-1051 aa) can form a complex with $\operatorname{Rad} 21(171-450$ aa) but not with $\operatorname{Rad} 21(1-172$ aa) or $\operatorname{Rad} 21$ (451-631 aa) (Figure 2B, middle panel). However, in the reverse co-purification, SA2 (1-1051 aa) does appear to form a complex with $\operatorname{Rad} 21$ (451-631 aa) (Figure 2B, right panel) but not with 
B

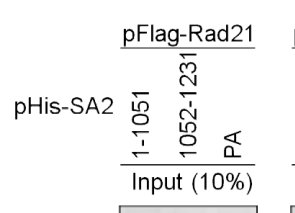

Flag-Rad21 - - -
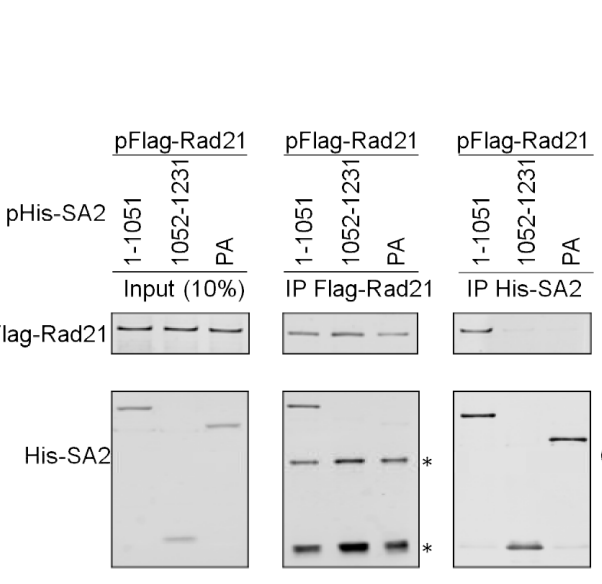

-

Flag pAb

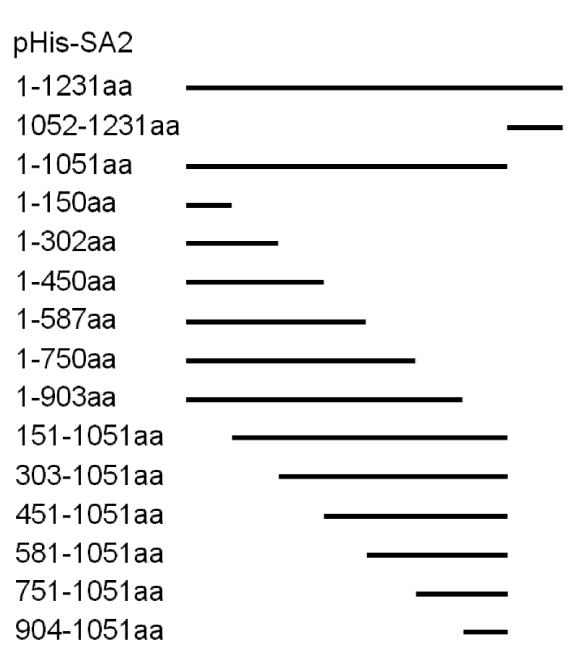

Illustration of pull down results

Flag-Rad21

His-SA2

IP His-SA2 IP Flag-Rad21

-

$++$

$-$

$++\quad++$

$+$

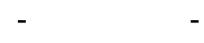

$+\quad+$

$++\quad+$

$++\quad+$

$+\quad+$

$++\quad+$

$+\quad+$

$+\quad++$

$---$
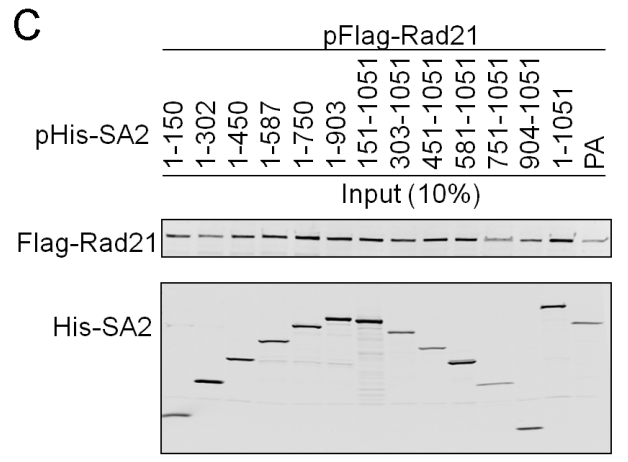

1223455678891011121314
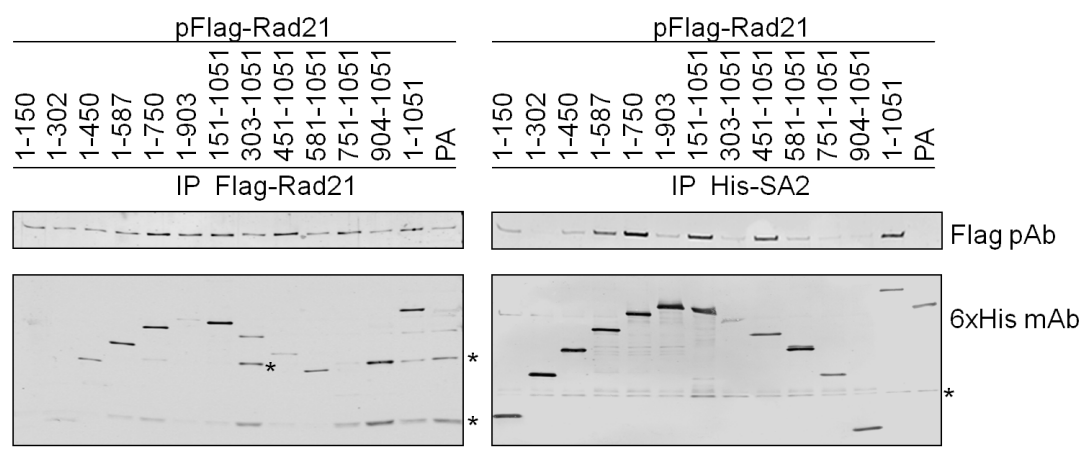

1516171819202122232425262728

2930313233343536373839404142

Figure 1. Characterization of the interaction between Rad21 and SA2 mutants. (A) Rad21 interacts with SA2 (1-1051 aa). Rad21 was coexpressed with either SA2 (1-1051 aa) or SA2 (1052-1231 aa) in Sf21 cells and co-purified by Ni-NTA or Flag beads. Rad21 co-expressed with the influenza A virus PA protein was used as a control. Western blot analysis was carried out using either the Flag polyclonal antibody (pAb) or the 6xHis monoclonal antibody (mAb). IgG bands are marked by asterisks $\left(^{*}\right)$. (B) Left panel shows the schematic illustration of the design of the SA2 deletion mutants used in (C). Right panel indicates the relative interaction strength of Rad21 and SA2 mutants in (C). ++: strong interaction; +: weak interaction; -: no interaction. (C) Flag-tagged Rad21 WT was co-expressed with His-tagged SA2 deletion mutants and co-purified by Ni-NTA or Flag beads. The influenza A virus PA was used as control. Nonspecific bands due to antibody cross-reaction are marked by asterisks $(*)$.

doi:10.1371/journal.pone.0069458.g001

Rad21 (1-172 aa). The discrepancy may be due to a non-specific in vitro interaction between SA2 and Rad21 (451-631) in His-SA2 IP contributed by overexpressed recombinant $\operatorname{Rad} 21(451-631)$ protein.

\section{SA2 and Rad21 form a stable complex}

To further investigate if the human SA2 directly binds to Rad21 and to validate the putative interacting domains of SA2 and Rad21 identified from the in vitro studies above, we co-expressed SA2 (1-1051 aa) and Rad21 (171-450 aa) in insect cells in which no other human cohesin proteins were present. The SA2 (11051 aa): $\operatorname{Rad} 21$ (171-450 aa) complex was purified using a NiNTA affinity column, followed by anion exchange and gel filtration (Figure 2C). The complex was eluted as a single peak in the gel filtration chromatography at a position earlier than SA2 (1-1051 aa) alone. Analytical ultracentrifugation was then performed for both the SA2 (1-1051 aa) and the SA2 (11051 aa):Rad21 (171-450 aa) complex. Velocity sedimentation data showed that the sedimentation coefficient of SA2 (1$1051 \mathrm{aa}): \operatorname{Rad} 21$ (171-450 aa) was indeed greater than that of SA2 (1-1051 aa) alone (Figure 2D), suggesting that SA2 (11051 aa):Rad21 (171-450 aa) can form a stable complex in vitro.
10 aa $\alpha$-helix on middle part of Rad21 interacts with SA2

To narrow down the polypeptide region of $\operatorname{Rad} 21$ that interacts with SA2, we utilized two approaches: 1) complex formation in the baculovirus expression system and 2) co-expression and immunoprecipitation (IP)-Western blot analysis in mammalian cells. Baculoviruses overexpressing progressive Rad21 deletion mutants with $~ 35$ amino acids increments/decrements from either the Nor C-terminus of the $\operatorname{Rad} 21$ middle region (171-450 aa) were generated (Figure 3A). SA2 (1-1051 aa) along with each of the Rad2 1 deletion mutants were expressed in Sf2 1 cells. The complex was purified using Ni-NTA or Flag beads and analyzed using Western blot. As shown in Figure 3B, $\operatorname{Rad} 21$ (351-450 aa), but not Rad21 (171-382 aa), could form a complex with SA2 (11051 aa) (Figure 3B, lanes 18 and 28 vs. lanes 12 and 22). While Flag-Rad2 1 (383-450 aa) co-purified His-SA2 (1-1051 aa) using Flag-beads (Figure 3B, lane 19), in the reciprocal IP, His-SA2 failed to co-purify with Rad21 (383-450 aa) (Figure 3B, lane 29), likely due to the low expression level of Flag-Rad21 (383-450 aa) (Figure 3B, lane 9). Collectively, the above results indicated that 351-450 aa of Rad21 is sufficient for its interaction with SA2, and $\operatorname{Rad} 21$ (383-450 aa) may be the minimal interacting region to 
A

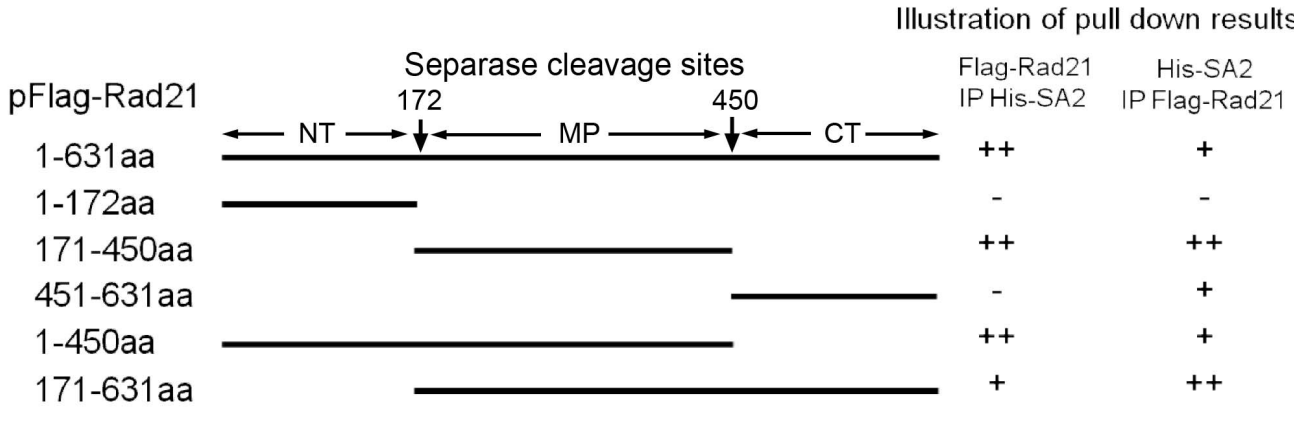

B

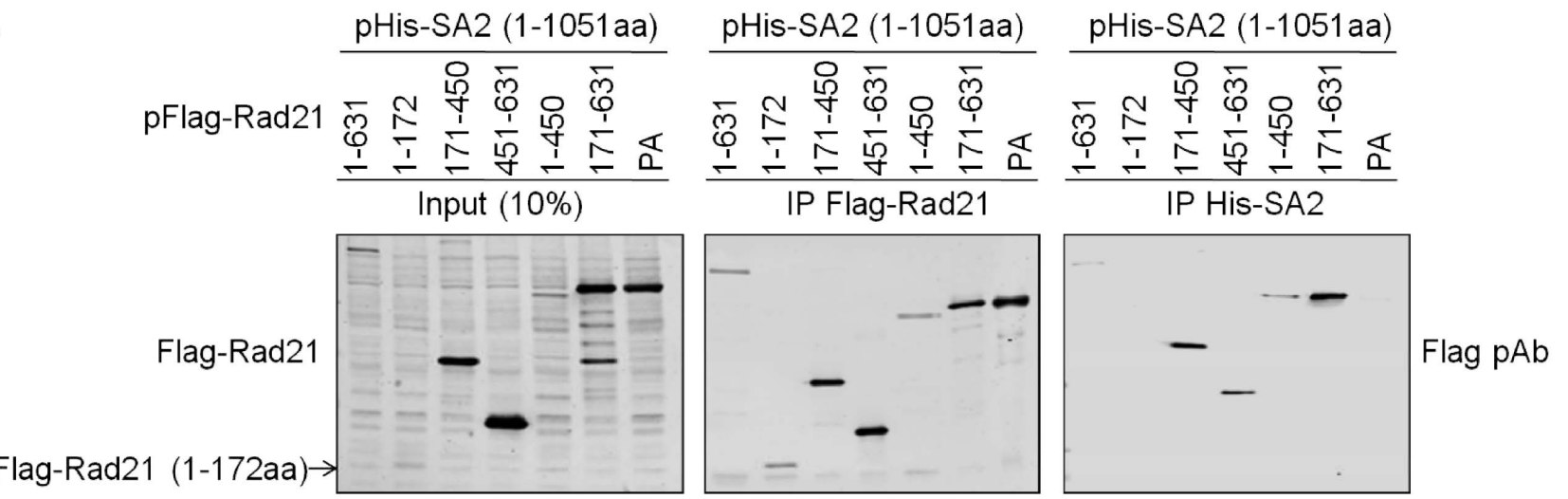

His-SA2 (1-1051 aa)
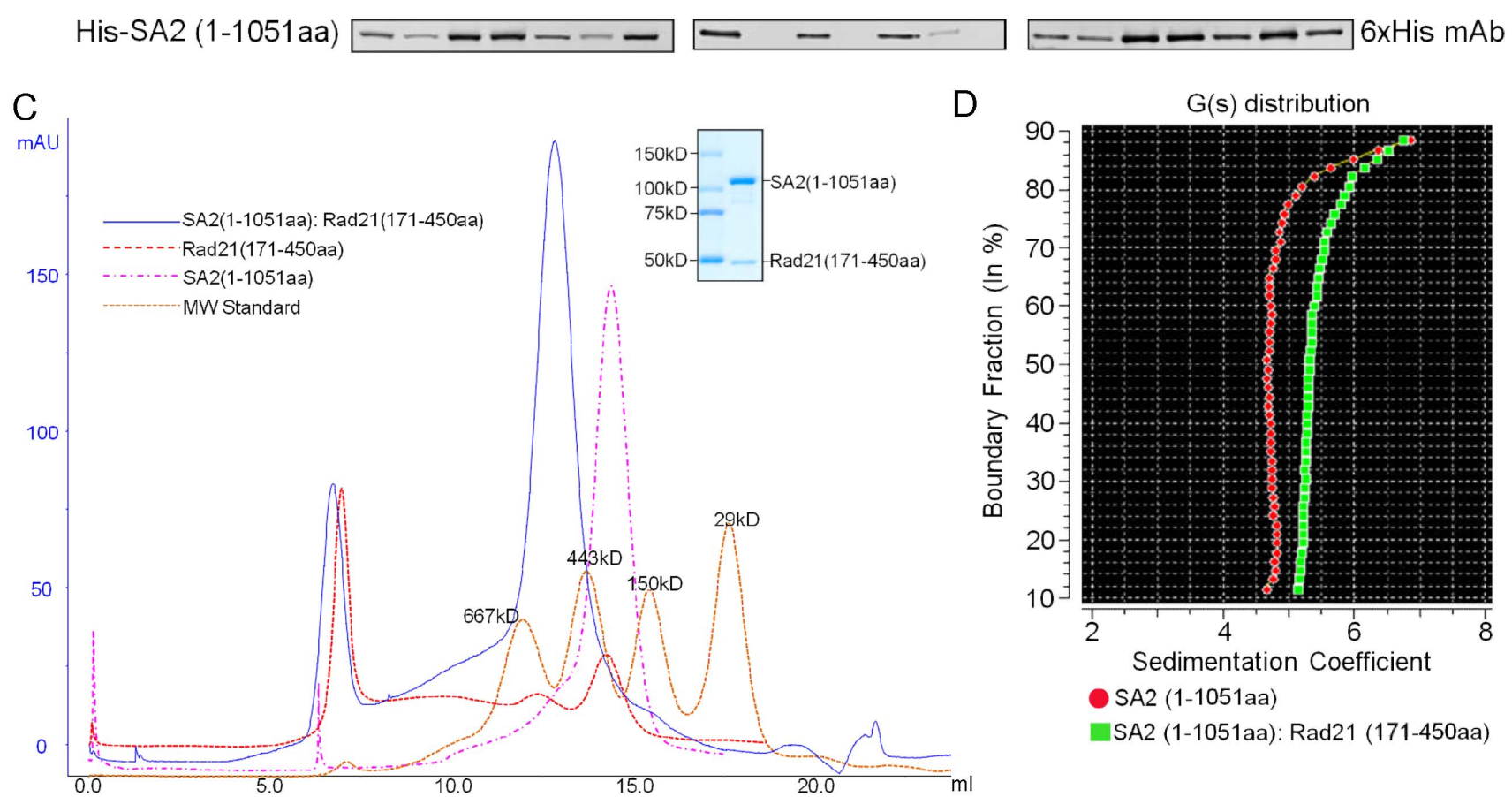

Figure 2. SA2 interacts with middle part of Rad21. (A) Schematic illustration of the Rad21 deletion constructs (left panel) and Rad21-SA2 interaction results from (B) (right panel). ++: strong interaction; +: weak interaction; -: no interaction. (B) Rad21 interacts with SA2 through the middle region. His-SA2 (1-1051 aa) was expressed along with Flag-Rad21 WT and deletion mutants and co-purified with Ni-NTA or Flag beads. SA2 (1-1051 aa) co-expressed with Flag tagged PA protein was used as a negative control. (C) Gel filtration chromatogram for the SA2 (1-1051 aa):Rad21 (171-450 aa) complex. SA2 (1-1051 aa) and Rad21 (171-450 aa) formed a stable complex. Inset shows the Coomassie-stained gel of the SA2:Rad21 complex purified by gel filtration. (D) Velocity sedimentation results for SA2 (1-1051 aa) and the SA2 (1-1051 aa): Rad21 (171-450 aa) complex. The complex shows an increase in the sedimentation coefficient compared to SA2 alone.

doi:10.1371/journal.pone.0069458.g002 
A

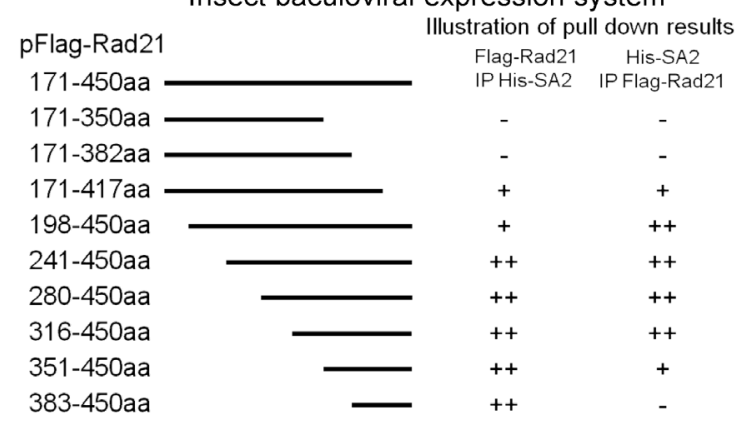

Mammalian expression system

Illustration of pull down results

$\begin{array}{lc}\text { Flag-Rad21 } & \text { Myc-SA2 } \\ \text { IP Myc-SA2 } & \text { IPFlag-Rad21 }\end{array}$

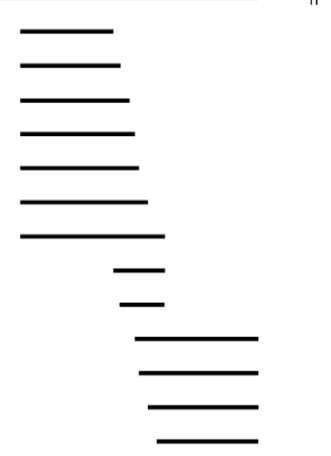

$\begin{array}{cc}\text { Myc-SA2 } & \text { IPFlag-Ra } \\ - & -\end{array}$

- -

- +

$++$

$+2+$

$++\quad++$

$\begin{array}{ll}++ & ++ \\ ++ & ++\end{array}$

$+\quad+$

$+$
C

pFlag Rad21
1-631aa
171-350aa
171-364aa
171-382aa
171-392aa
171-400aa
171-417aa
171-450aa
351-450aa
365-450aa
393-631aa
401-631aa
418-631aa
436-631aa

$+$

B

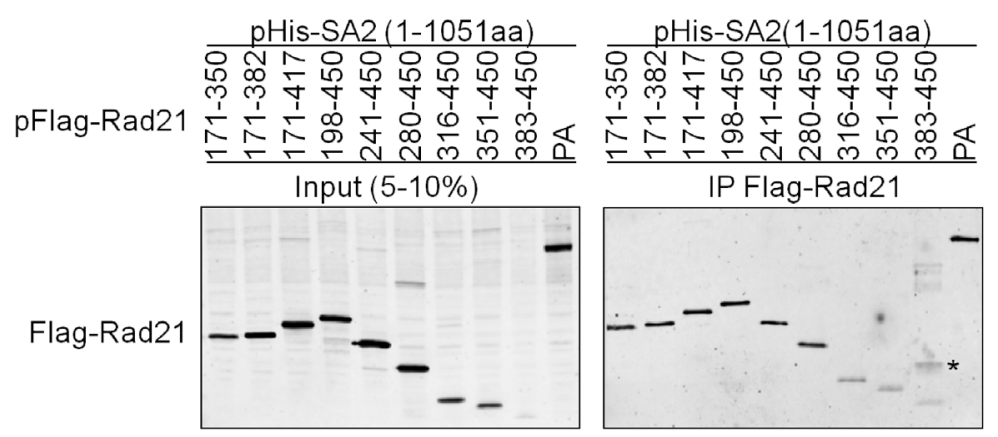

pHis-SA2 (1-1051aa)

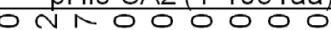

ñ

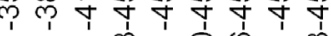

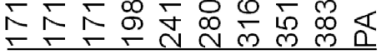

IP His-SA2

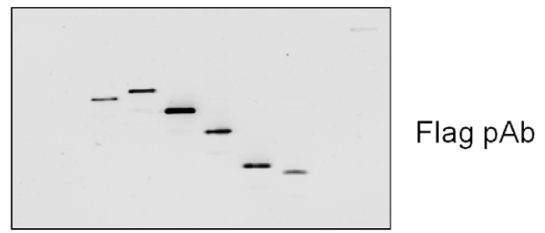

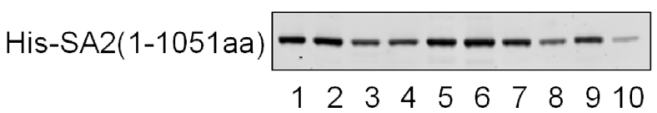

$\cdots \cdots-1$

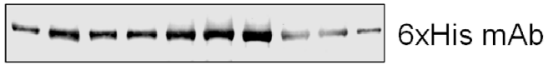

$\begin{array}{llllllllll}1 & 2 & 3 & 4 & 5 & 6 & 7 & 8 & 9 & 10\end{array}$

11121314151617181920

21222324252627282930

D

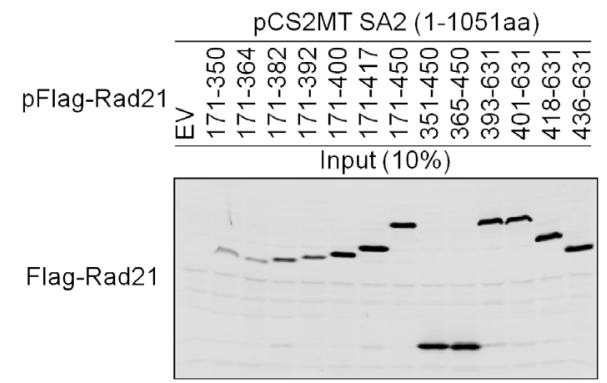

pCS2MT SA2 (1-1051aa)

PCS2MT SA2 (1-1051aa)

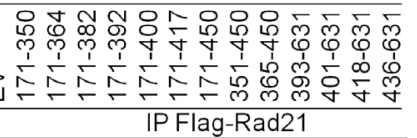

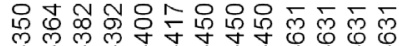

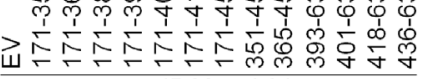

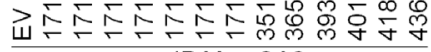

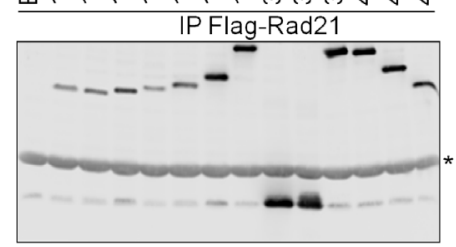

IP Myc-SA2

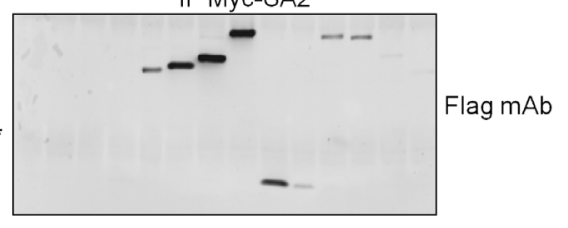

$----1-$

- - - - - - - Myc pAb

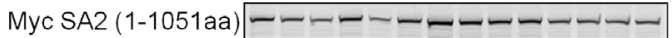

12234556788991011121314

1516171819202122232425262728

2930313233343536373839404142

Figure 3. SA2 interacts with a 10 aa region of middle part Rad21. (A) Schematic illustration of the middle portion Rad21 deletion constructs made in the baculovirus system and interaction results of Rad21-SA2 from (B). ++: strong interaction; +: weak interaction; -: no interaction. (B) Rad21 (171-382 aa) does not interact with SA2. His-SA2 (1-1051 aa) was expressed along with the Flag tagged Rad21 deletion mutants and co-purified with Ni-NTA or Flag beads. Antibody cross-reaction bands are marked by asterisk $\left(^{*}\right)$. (C) Schematic illustrations of Rad21 deletion constructs in the context of the full length Rad21 in the mammalian expression vector pFlag CMV2 and interaction results from (D). ++: strong interaction; +: weak interaction; -: no interaction. (D) Rad21 383-392 aa region is critical for interacting with SA2. Myc-SA2 (1-1051 aa) was co-transfected along with the Flag-Rad21 deletion mutants and immunoprecipitated with Flag or Myc beads and probed with either the Myc polyclonal antibody (Myc pAb) or the FLAG mAb. Flag empty vector (EV) was used as a negative control.

doi:10.1371/journal.pone.0069458.g003

form a Rad21-SA2 complex in vitro in this series of deletions using insect cells.

To further narrow down the interacting domain of $\operatorname{Rad} 21$ with SA2, we made another set of Rad21 constructs with deletion at the 383-450 region (Figure 3C) and validated the interaction of Rad21 and SA2 using a mammalian cell expression system. SA2(1-1051 aa) was cloned into the pCS2MT vector with a $6 \mathrm{xMyc}$ epitope at the N-terminus, and a series of $\operatorname{Rad} 21$ mutants were cloned into the pFlag CMV2 vector carrying a Flag epitope at the N-terminus (Figure 3C). SA2 (1-1051 aa) was transfected into $293 \mathrm{~T}$ cells along with each of the Rad21 deletion constructs. Co-IP with Flag beads and Myc monoclonal antibody (mAb)conjugated agarose beads (Myc beads) were then used to analyze the interactions between SA2 and the Rad21 mutants (Figure 3D). Co-IP results showed that Rad21 (171-392 aa), but not the Rad21 (171-382 aa) or Rad21 (393-631 aa), were able to pull down SA2 
(1-1051 aa) (Figure 3D, lane 19 vs 18 and 25; lane 33 vs 32), indicating that amino acids 383-392 are critical for the Rad21SA2 interaction in human cells.

A secondary structure prediction for $\operatorname{Rad} 21$ (171-450 aa) using the program PredictProtein [23] revealed that the entire region is largely unstructured/disordered with only three $\alpha$-helices, among which only two were predicted with high probability (Figure S2). Interestingly, one of these two highly probable $\alpha$-helices is formed by 383-392 aa. An alignment of the $\operatorname{Rad} 21$ proteins from various vertebrate species indicated the presence of several conserved amino acid residues, including $\mathrm{L}^{385}, \mathrm{~F}^{389}$, and $\mathrm{T}^{390}$ (Figure 4A). To confirm that 383-392 aa are critical for the Rad21-SA2 interaction and to identify the amino acids that are essential for this interaction, a $\operatorname{Rad} 21$ deletion mutant $\operatorname{Del}(383-392$ aa) and several site-directed mutants were generated (Figure 4B). After Flag-Rad21 and Myc-SA2(1-1051 aa) were expressed in $293 \mathrm{~T}$ cells, co-IP results confirmed that the full-length $\operatorname{Rad} 21$ devoid of 383-392 aa failed to pull down SA2 (1-1051 aa) (Figure 4B, lanes 22 and 34 ). The mutation of two conserved amino acids $\mathrm{L}^{385}$ and $\mathrm{F}^{389}$ to alanine (A) within this 10 aa stretch in Rad21 significantly reduced its interaction with SA2 (Figure 4B, lanes 18 and 30). The combined mutations of all three conserved residues $\mathrm{L}^{385}, \mathrm{~F}^{389}$, and $\mathrm{T}^{390}$ to alanine was able to abrogate the interaction between $\operatorname{Rad} 21$ and SA2 (Figure 4B, lanes 21 and 33), indicating that the three conserved amino acids $\mathrm{L}^{385}, \mathrm{~F}^{389}$, and $\mathrm{T}^{390}$ are essential for the Rad21-SA2 interaction ex vivo. This result is consistent with an earlier report that the region 362-403 aa of $\operatorname{Rad} 21$ interacts with SAl [22].

\section{Interaction of SA-binding deficient Rad21 mutants and other cohesin subunits}

To investigate if SA2-binding deficient $\operatorname{Rad} 21$ mutants fail to co-immunoprecipitate endogenous SA2, Flag-Rad21 WT or mutants were expressed in $293 \mathrm{~T}$ cells and immunoprecipitated. Co-IP of endogenous cohesin subunits was analyzed using Western blot. In contrast to Rad21 WT and the single mutant (SM) L385A (Figure 5A lane 8-9), Rad21 double mutant (DM) L385A F389A, triple mutant (TM) L385A F389A T390A and deletion mutant
[Del(383-392 aa)] significantly reduced the co-IP of endogenous SA1 and SA2 (Figure 5A, lane 10-12, Figure S3A). To further confirm that the interaction of SAl and Rad21 is indeed disrupted by SA2-binding deficient Rad21 mutants, $293 \mathrm{~T}$ cells were cotransfected with Myc-SAl and Flag-Rad21 constructs, and co-IP results verified that the Flag-Rad21 mutants significantly reduced the interaction with SA1 (Figure S4). Reduction of both SAl and SA2 co-immunoprecipitation by Rad21 DM, TM, and Del mutants suggests that SA1 and SA2 bind to the same region of Rad21. Thus, we call Rad21 383-392 aa region as MP SAbinding motif because this region resides on the MP of Rad21 after $\operatorname{Rad} 21$ is cleaved by Separase at R172 and R450 (Figure 2A).

To examine if the disruption of the $\operatorname{Rad} 21-\mathrm{SA} 1 / 2$ interaction has any effect on the association of $\operatorname{Rad} 21$ with other cohesin core subunits, the IP products pulled down by Flag-tagged Rad21 proteins from the above experiments were analyzed using antibodies against other cohesin core subunits and associating proteins. The immunoprecipitation of both Smcl and Smc3 remained unchanged in all the $\operatorname{Rad} 21$ mutants compared to the WT control (Figure 5A, S3A), indicating that the disruption of the Rad21-SA2 interaction has no apparent effect on the Smc1-Smc3Rad21 interaction. Co-IP of cohesin-associated factor Wapl was also not affected by the MP SA-binding deficient $\operatorname{Rad} 21$ mutations (Figure 5A). The effect of the MP SA-binding deficient Rad21 mutations on Pds5 association could not be clearly judged in this experiment because both Pds5A and Pds5B were not coimmunoprecipitated well (Figure 5A). However, Figure 6E (lane 7 vs. 8) demonstrated co-IP of Pds5 was not disrupted by the mutations on the MP SA-binding motif of Rad21.

To confirm the Rad21 mutants can be incorporated into the cohesin complex and associated with chromosomes, we knocked down the endogenous Rad21 with siRNA against 3'-UTR of Rad21, expressed WT or mutant Rad21 in $293 \mathrm{~T}$ cells, and performed IP from chromatin fractions. The chromatin fraction without the contamination of soluble fraction was validated with GAPDH and tubulin monoclonal antibodies (mAb) (Figure 5B). The immunoprecipitation of Smc3 by all the Rad21 mutants in chromatin fractions was not affected (Figure 5C). Because Rad21

A

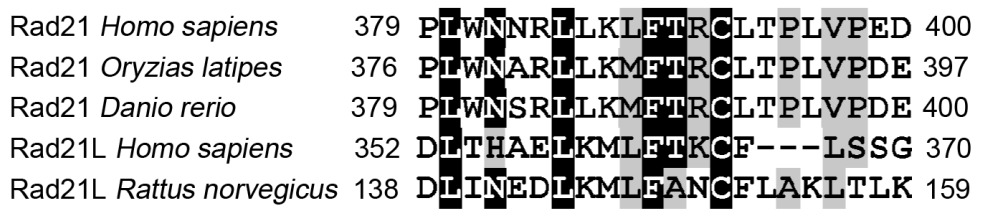

B
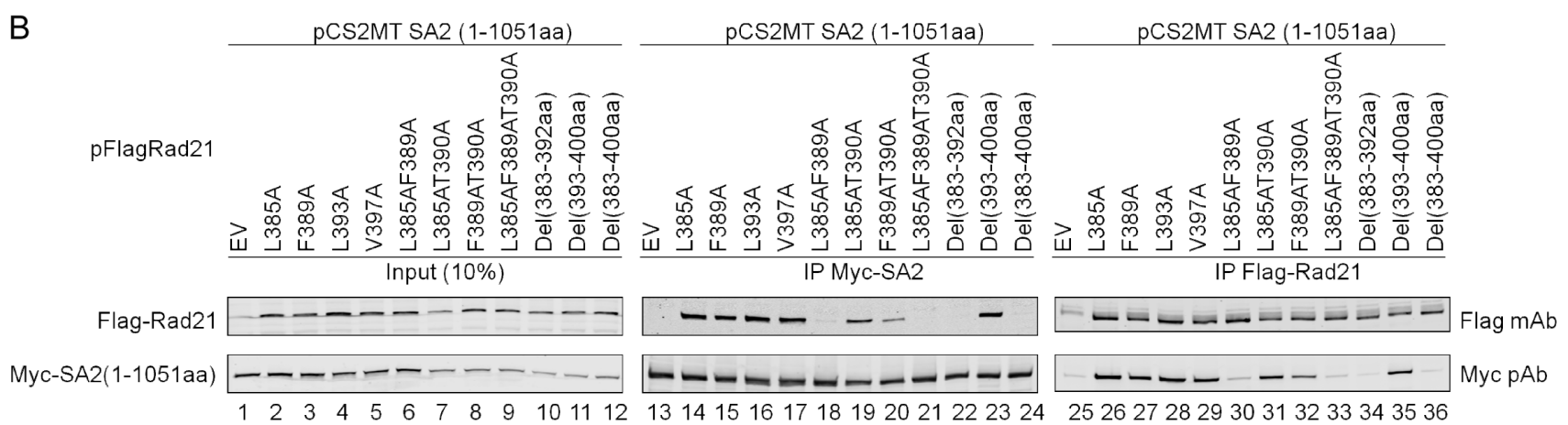

Figure 4. $\mathbf{L}^{\mathbf{3 8 5}}$ and $\mathbf{F}^{\mathbf{3 8 9}}$ are critical for Rad21 to interact with SA2. (A) Sequence alignment of Rad21 from various vertebrate species. The sequence alignment was prepared using BioEdit (http://www.mbio.ncsu.edu/bioedit/). The conserved Rad21 $\mathrm{L}^{385}, \mathrm{~F}^{389}$ and $\mathrm{T}^{390}$ residues were used for making site-directed mutations. (B) Co-immunoprecipitation analysis indicating the importance of $\mathrm{L}^{385}$, $\mathrm{F}^{389}$, and $\mathrm{T}^{390}$ residues on Rad21 for its interaction with SA2. Flag-Rad21 and Myc-SA2(1-1051 aa) were expressed in 293 T cells. Co-IP was performed $48 \mathrm{~h}$ after transfection. doi:10.1371/journal.pone.0069458.g004 

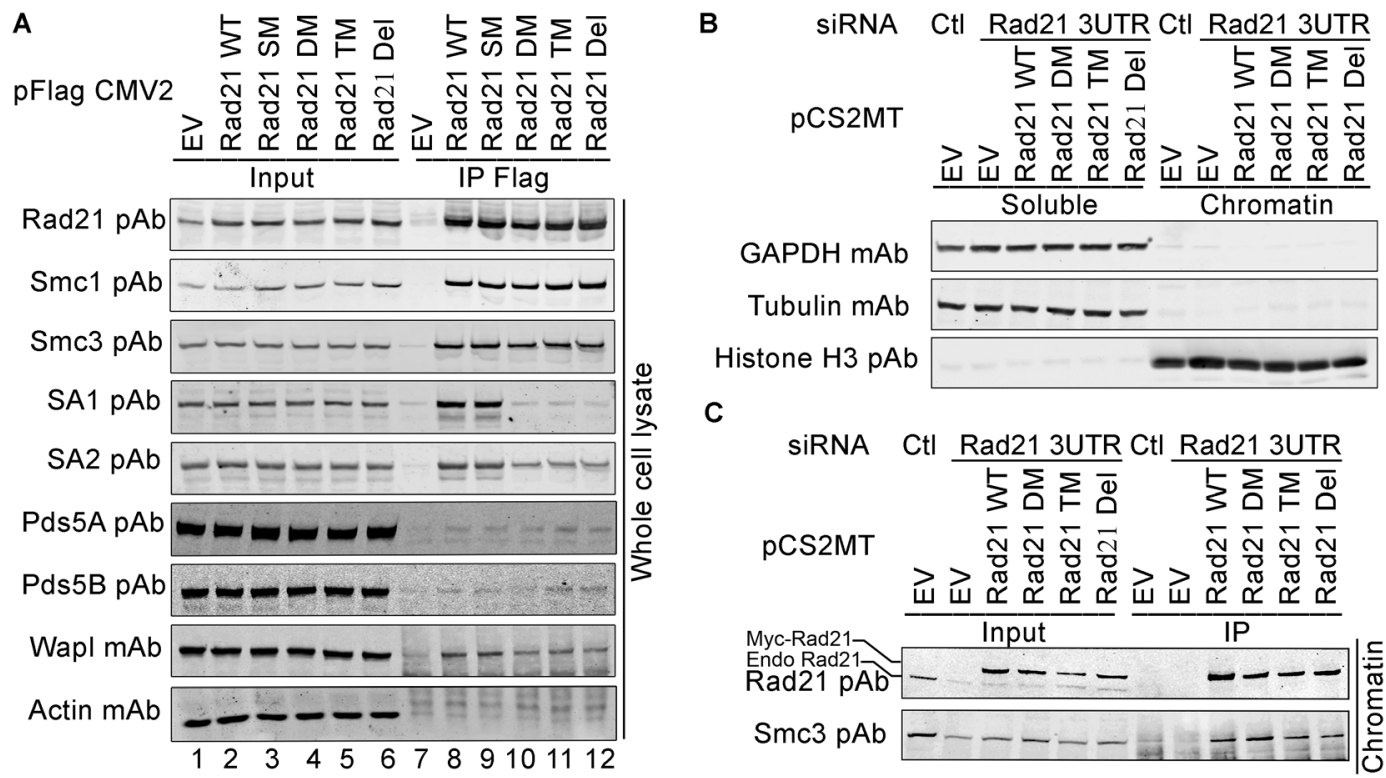

Figure 5. Co-immunoprecipitation of other cohesin subunits by Rad21 with mutations or deletion on MP SA-binding motif. (A) 293 T cells were transfected with pFlag CMV2 Rad21 WT or mutants and co-immunoprecipitation was performed using whole cell lysate. Cells transfected with empty vector (EV) was used as control. Immunoblotting shows the cohesin core subunits and associating proteins immunoprecipitated by Rad21 WT and mutants. (B-C) 293 T cells were transfected with pCS2MT Rad21 WT or mutants. EV was used as control. Endogenous Rad21 was knocked down with Rad21 3'-UTR siRNA. Scrabbled siRNA was used as control (Ctr). Proteins from chromatin fraction were isolated and used for IP. Immunoblotting shows the chromatin fraction was not contaminated by the soluble fraction (B) and like WT Rad21, mutant Rad21 was found on chromatin and co-immunoprecipitated by cohesin-Smc3 (C). EV: empty vector, WT: wild type, DM: L385A T390A, TM, L385A F389A T390A, Del: del(383-392 aa).

doi:10.1371/journal.pone.0069458.g005

binding to Smcl is required for its binding to Smc3 [25], the results confirmed that the Smcl-Smc3-Rad21 complex is not only present in soluble fraction, but it is also capable of binding to chromatin.

\section{$\mathrm{N}$-terminus of Rad21 contains a second SA-binding site}

We noticed that mutation or deletion of the SA-binding motif at 383-392 aa of Rad21 could not completely abrogate the interaction of SA1 and SA2 in the co-IP experiments (Figure 5A, $\mathrm{S} 3 \mathrm{~A})$. We reasoned that there may be additional SA-binding site/s on Rad21. Using a set of pilot experiments in $293 \mathrm{~T}$ cells, we found that SA1 and SA2 could be co-immunoprecipitated by the N-terminal fragment (1-172 aa) of $\operatorname{Rad} 21(\operatorname{Rad} 21 \mathrm{NT})$ and the middle part (173-450 aa) of $\operatorname{Rad} 21(\operatorname{Rad} 21 \mathrm{MP})$, but not the Cterminal fragment (451-631 aa) of $\operatorname{Rad} 21(\operatorname{Rad} 21 \mathrm{CT})$ (Figure S5). As described above, we have identified 383-392 aa as one of the SA-binding motifs on $\operatorname{Rad} 21 \mathrm{MP}$, and because there is no interaction of SAl/2 within the C-terminal (451-631 aa) domain of $\operatorname{Rad} 21$, we focused our analysis for a possible SA-binding motif on the N-terminal domain of $\operatorname{Rad} 21(\operatorname{Rad} 21 \mathrm{NT})$. We made deletion mutants with 20 aa increments from the N-terminus of Rad21 (1-450 aa), with triple mutations (TM) of L385A-F389AT390A on the identified MP SA-binding motif (Figure 6A). The Rad21 proteins were expressed in $293 \mathrm{~T}$ cells, and IP was performed. The immunoblotting results showed that deletion of the first 40 aa of $\operatorname{Rad} 21$ (41-450 aa) reduced the co-IP of SA2, and deletion of first 80 aa (Rad21 81-450) completely abolished the co-IP of SA2 (Figure 6B). Because these $\operatorname{Rad} 21$ constructs contain triple mutations at L385F389T390 along with the deletions of C-terminus (451-631 aa) and a portion of the Nterminus of $\operatorname{Rad} 21$, the interaction with $\mathrm{SA} 2$ in the co-IP experiments was significantly decreased. The above data suggest that another SA2 interacting motif is located within the first 80 aa of the Rad21 NT.

First 150 aa of the N-terminus of $\operatorname{Rad} 21$ are highly conserved across different species (Figure 6C). By examining the amino acid sequence around 60 aa, we speculated that the region of 61-80 aa of Rad21 may be critical for SAl/2 interaction. Deletion of $61-$ 80 aa region of $\operatorname{Rad} 21$ (1-450 aa) TM indeed blocked the co-IP of $\mathrm{SAl} / 2$ (data not shown). To further confirm these two SA-binding motifs (61-80 aa, 382-392 aa) in full-length $\operatorname{Rad} 21$, we made three full-length constructs with quadruple mutations (QM) of R69A, K70A, K72A and Y73A at the region of 61-80 aa and TM of L385A, F389A and T390A at the region of 383-392 aa either alone or in combination (Figure 6D). $\operatorname{Rad} 21$ mutants were expressed in $293 \mathrm{~T}$ cells and immunoprecipitated with Flag mAb conjugated agarose beads. Immunoblotting results demonstrated that the efficiency of co-immunoprecipitation of $\mathrm{SA} 1 / 2$ by Rad2 1 constructs is as follows: $\operatorname{Rad} 21 \mathrm{WT}<\operatorname{Rad} 21 \mathrm{QM}<\operatorname{Rad} 21 \mathrm{TM}$, and Rad21 QM+TM failed to pull down SA1/2 (Figure 6E). Similar to the TM, the QM did not affect the co-IP of Smcl and Smc3. However, unlike the Rad21 WT or the Rad21 TM, the Rad21 QM reduced the co-IP of Pds5 (Figure 6E). Based on the above data, we conclude that at least two SA-binding sites are on $\operatorname{Rad} 21$ : one is located on the N-terminus, and the other is on the MP of Rad21 molecule. Similar to the MP SA-binding motif, we name the SA-binding domain on the N-terminus of Rad21 the NT SA-binding motif.

Mutations on SA-binding motifs of Rad21 reduce Rad21Rad21 dimerization and result in premature separation of sister chromatids

Previously, we proposed the handcuff model of cohesin configuration, in which we suggested the anti-parallel dimerization 
A

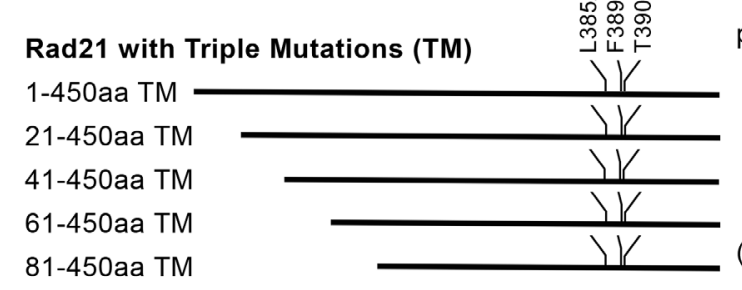

C

Human 41 VESIISPKVK-MÁLRTSGHLLL'GVVRIYHRKÁKYLLADCNEÁFIKIKMAFR 90

Mouse 41 VESIISPKVK-MALRTSGHLLLGVVRIYHRKAKYLLADCNEAFIKIKMAFR 90

Xenopus 41 VESIISPKVK-MALRTSGHLLLGVVRIYHRKAKYLLADCNEAFIKIKMAFR 90

Fly 41 VESIISPKVK-LALRTSGHLLLGVVRIYSRKAKYLLADCNEAFVKIKMAFR 90

pombe 41 VHAIVTEETAPMALRLSGQLMLGVVRIYSRKARYLLEDCTEALMRLKMSFQ 91

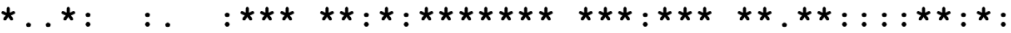

D

$\operatorname{Rad} 21$

WT

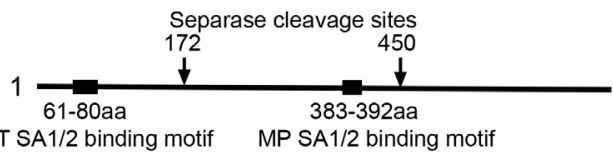

Quadruple

Mutations

(QM)

TM
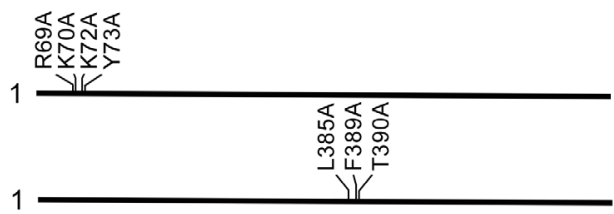

$\mathrm{QM}+\mathrm{TM}$

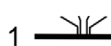

采
631 aa

631 aa

631 aa
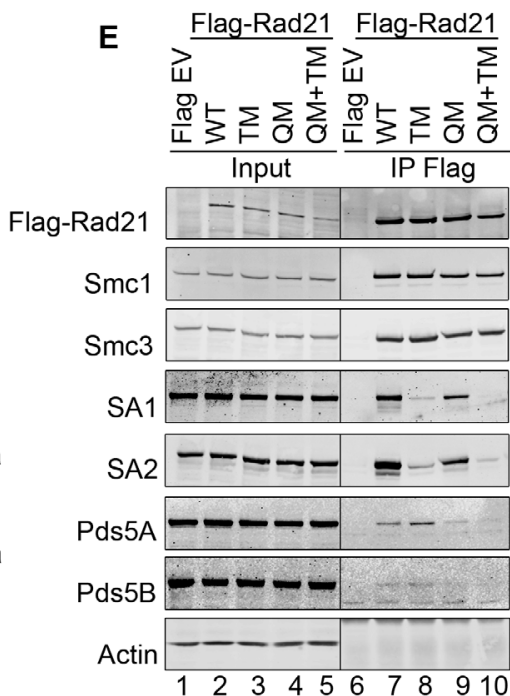

Figure 6. Identification of SA-binding site on N-terminus of Rad21. (A) Schematic illustration shows the N-terminal truncated mutants of Rad21 (1-450 aa) with triple mutations (TM) on middle part of SA-binding motif. The CDNA was cloned into pFlag CMV2 vector. (B) Immunoblotting of SA2 co-IP by Rad21 (1-450 aa) WT and mutants. 293 T cells were transfected with the constructs shown in (A) and IP was performed $40 \mathrm{~h}$ after transfection. Deleting the first 80 aa of Rad21 (1-450 aa) TM inhibits the co-IP of SA2. The second and third panels are from the same blot. The third panel was enhanced for better visualization. (C) Clustal format alignment of Rand21 (41-90 aa) by MAFFT L-INS-i (v7.015b). The bottom line shows the conserved amino acids from fission yeast (S. pombe) to human Rad21. Invariant, conserved, and semi-conserved residues are indicated by an asterisk (*), colon (:), and period (.), respectively. (D) Schematic illustration shows the full length Rad21 and the mutations on the two SA-binding motifs located on the NT and MP of Rad21, respectively. The CDNAs of Rad21 WT and mutants with quadruple mutations (QM) on the NT SA-binding motif and/or triple mutations (TM) on the MP SA-binding motif were cloned into pFlag CMV2 vector. (E) Immunoblotting of co-IP of cohesin subunits by Rad21 WT or mutants. The dividing lines indicate that interfering lanes have been spliced out.

doi:10.1371/journal.pone.0069458.g006

of $\operatorname{Rad} 21$ molecules through SA1/2. However, we did not rule out possible roles of other cohesin factors in the Rad21-Rad21 dimerization [17]. To test if mutations on SA-binding motifs of Rad21 disrupt Rad21-Rad21 dimerization, we performed co-IP of Flag and Myc epitope-tagged Rad21 molecules with SA-binding motif mutants. Immunoblotting results showed that mutation or deletion of the SA-binding motif at MP of $\operatorname{Rad} 21$ alone did not significantly reduce the Rad21-Rad21 interaction (Figure S3B). Myc-tagged Rad21 with mutation or deletion of the MP SAbinding motif could also co-immunoprecipitate endogenous Rad21 (Figure S3A). Similar results were observed with mutation or deletion of the SA-binding motif at N-terminus of Rad21 alone (data not shown). However, compared to wild type Rad21, the coIP of Flag-Rad21 and Myc-Rad21 with mutations on both NT and MP SA-binding motifs (QM+TM) of Rad21 was significantly reduced (Figure 7A, lanes $5 \& 6,8 \& 9$ ), suggesting that $\mathrm{SAl} / 2$ links Rad21 proteins at multiple sites and also play an important role in forming the two-ring cohesin complex.

To examine the physiological consequence on the chromosomal cohesion after the interaction of $\mathrm{SA} 1 / 2$ and $\operatorname{Rad} 21$ is disrupted, Myc-tagged WT and mutant Rad21 were expressed in $293 \mathrm{~T}$ cells. Myc empty vector (EV) was used as a control. To examine the effect of the ectopic protein, endogenous $\operatorname{Rad} 21$ was knocked down using Rad21 3'-UTR siRNA. Compared to the samples treated with control siRNA, at least $80 \%$ of endogenous Rad21 protein in samples treated with Rad21 3'-UTR siRNA was knocked down (Figure 7B). The expression of WT and mutant Myc-tagged $\operatorname{Rad} 21$ was equivalent (Figure 7B). Metaphase 
A

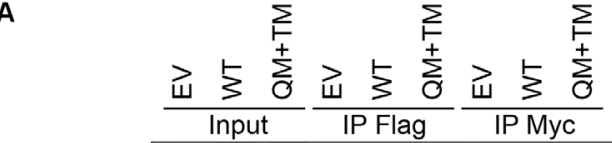

$\operatorname{Rad} 21 \mathrm{mAb}$
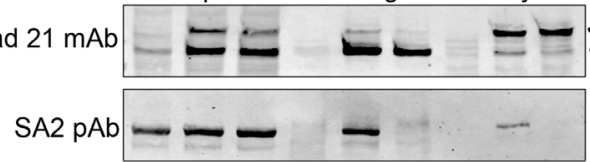

Actin $\mathrm{mAb}$
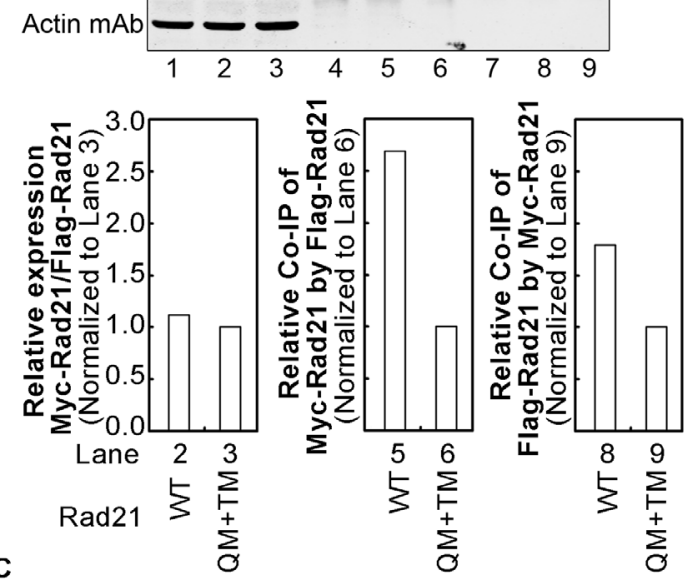

B

B Myc-Rad21

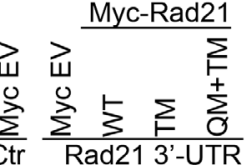

-Flag-Rad21

IEndo Rad21

Rad21 pAb

Actin $\mathrm{mAb}$

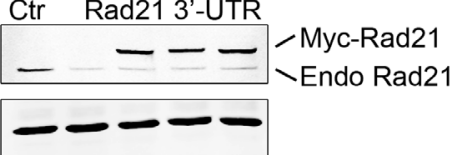

D

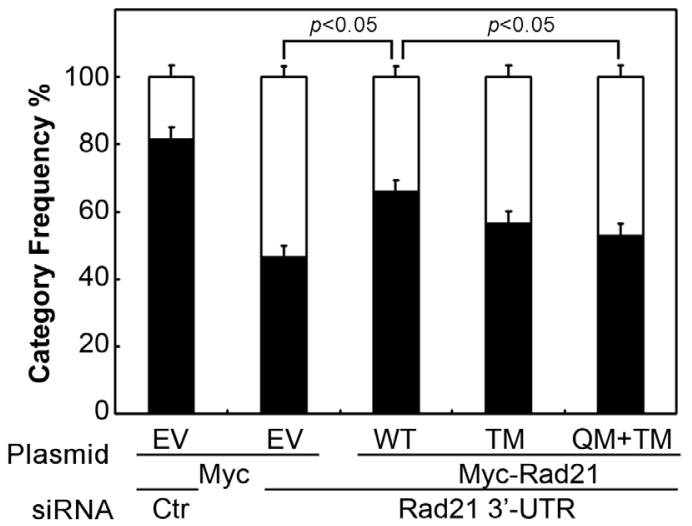

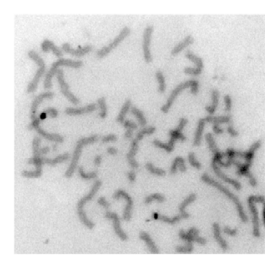

Arm Cohesed

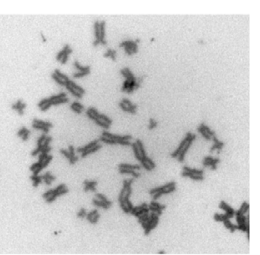

Arm Separated

Cohesed Chromosomes

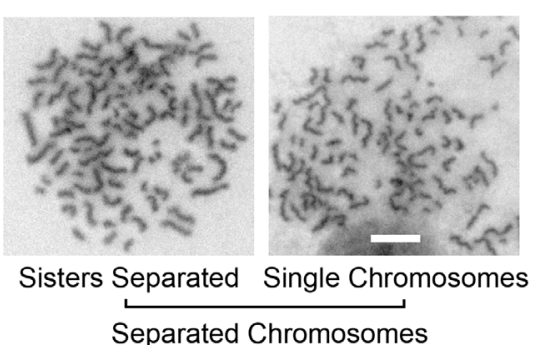

Separated Chromosomes

Figure 7. Reduced Rad21-Rad21 dimerization and defect in sister chromatid cohesion in cells expressing Rad21 with mutations on SA-binding motifs. (A) Co-IP of differentially tagged Rad21 with mutations on both NT and MP SA-binding motifs. Rad21 WT or mutants of QM+TM (see Figure 6D) were tagged with Flag or Myc on its N-terminus and expressed in $293 \mathrm{~T}$ cells. IP was performed with Flag mAb or Myc pAb conjugated agarose beads and immunoblotting was carried out. The bar graphs show the relative expression or co-IP level of Myc-Rad21 and FlagRad21. Rad21 WT bands were normalized using respective Rad21 QM+TM mutant bands. (B-D) Rad21 mutants cannot rescue the premature separation of sister chromatids caused by Rad21 knockdown. 293 T cells were treated with Rad21 3'-UTR siRNA or a control (Ctr) siRNA for $24 \mathrm{~h}$ before Myc-Rad21 WT or mutant plasmids was transfected. The knockdown of endogenous Rad21 and the expression of Myc-Rad21 were analyzed by Western blot using Rad21 pAb (B). Metaphase chromosome spread was performed and the status of sister chromatid cohesion was analyzed according to the categories shown in (C). About 100 mitotic cells were counted for each treatment. The frequency of mitotic cells was calculated and plotted in (D). EV, empty vector; WT, wild Type; QM, quadruple mutant R69A K70A K72A Y73A; TM, triple mutant L385A F389A T390A. Bar size $=10 \mu \mathrm{m}$. Values were compared using Student's $t$-test.

doi:10.1371/journal.pone.0069458.g007

chromosome spread analysis was performed, and mitotic cells from each sample were counted according to their separation status: 1) cohesed chromosomes that include chromatids linked at the centromeres with arm cohesed or separated and 2) separated chromosomes that include chromatids separated but still in alignment with each other and single chromatids randomly scattered (Figure 7C). Approximately $20 \%$ of the control siRNA treated cells showed separated chromatids. In comparison to the control siRNA treated cells, approximately $55 \%$ precocious sister chromatid separation was noted in the cells treated with Myc EV control plus Rad21 3'-UTR siRNA, the phenotype of which could be partially but significantly $(\mathrm{p}<0.05)$ rescued $(\sim 20 \%)$ by overexpressing the ectopic WT $\operatorname{Rad} 21$ (Figure 7D). However, Rad21 construct with mutations on both the NT and MP SAbinding motifs (QM+TM) failed to significantly rescue the PCS phenotype caused by the Rad21 knockdown (Figure 7D). These results suggest that both the NT and MP SA-binding motifs of $\operatorname{Rad} 21$ are not only required for Rad21-SA2 interaction but also are physiologically important for sister chromatid cohesion.

\section{Discussion}

It has been reported that SA2 associates with the tripartite cohesin ring through binding to Rad21 [5]. However, details of the Rad21-SA2 interaction at the molecular level and its consequence in chromosomal segregation are lacking. Here we show that the 301-750 aa region of SA2 interacts with Rad21. We have also identified two SA-binding motifs encompassing 6180 aa and 382-292 aa regions of Rrad21. Deletion or mutations of these $\operatorname{Rad} 21$ motifs result in the failure of $\operatorname{Rad} 21-\operatorname{Rad} 21$ dimerization and precocious chromatid separation. 


\section{SA1 and SA2 interact with Rad21 in a similar way}

Using the insect cell expression system, we identified a broad region (301-750aa) of SA2 that interacts with $\operatorname{Rad} 21$. It is possible that a different portion of SA2 interacts with a different region of $\operatorname{Rad} 21$, which is evident from our studies in mammalian cells in which Rad2 1 contains at least two SA-binding motifs. In metazoa, phosphorylation of SA2 by Polo-like kinase 1 (Plkl) at the chromosome arms during prophase is required for the dissolution of arm cohesion [26-28]. The C-terminal SA2 region (residues 1052-1231aa) contains 12 of the 14 SA2 phosphorylation sites, including the seven highly phosphorylated sites [29]. An interesting note is that this C-terminal SA2 region is not required for SA2 binding to the SA-binding motif of $\operatorname{Rad} 21$, suggesting its regulatory function.

SA1, the other Scc3 ortholog in humans, contains 1258 amino acid residues and shares approximately $70 \%$ sequence identity with SA2. Most of the amino acid variations between SA1 and SA2 are in the 1-68 aa and 1075-1162 aa regions. SA1 shares $77 \%$ sequence identity with SA2 at the 301-751 aa region of SA2 that interacts with $\operatorname{Rad} 21$. The $301-751$ aa region in SA2 also contains stromalin conserved domain (SCD), which is conserved in SA1, SA2, and SA3 [30], suggesting that similar molecular interactions may exist between SA1 and Rad21. Our results from mammalian cells show that the Rad21 mutants with reduced interaction with SA2 also decrease the interaction with SAl, indicating SA1 and SA2 interact physically with $\operatorname{Rad} 21$ in a similar way. The physiological significance of the 301-751 aa region in SA2 is also demonstrated by the recent findings of truncated and missense mutations in this region in a number of human cancers [20].

\section{Rad21 has two SA-binding motifs}

$\mathrm{SAl} / 2$ is known to interact with cohesin via $\operatorname{Rad} 21$, but which portion of Rad21 binds to SAl/2 is not clear. According to the two Separase cleavage sites on human $\operatorname{Rad} 21$, we made three Rad21 constructs containing the NT, MP, and CT fragment, respectively, to map which part of Rad21 interacting with SAl/2. Our results show that in the insect cell expression system, only the MP of Rad21 can interact with SA2, whereas in mammalian cells both the NT and the MP fragments of $\operatorname{Rad} 21$ can coimmunoprecipitate SA2. However, the Rad21 CT cannot interact with $\mathrm{SAl} / 2$ in either system. Further studies indicate that the interaction between the MP of $\operatorname{Rad} 21$ and SA2 is restricted to a 10 aa $\alpha$-helical region. In this 10 aa $\alpha$-helical region, $L^{385}$ and $F^{389}$ are next to each other in the helix (Figure S6) and Rad21-SA1/2 interaction is disrupted when $\mathrm{L}^{385}$ and $\mathrm{F}^{389}$ are mutated into alanine, suggesting that this particular side of the $\alpha$-helix interacts with $\mathrm{SAl} / 2$.

The Rad21 NT can co-immunoprecipitate endogenous Rad21 in $293 \mathrm{~T}$ and HeLa cells, but it fails to interact with SA2 in the insect cell expression system. Two possibilities explain this disparity. One is that $\mathrm{SAl} / 2$ interaction with $\operatorname{Rad} 21$ NT requires additional factors that mammalian cells, but not insect, cells have. One or more of cohesin-associated proteins, such as Pds5 and Sororin, are probable candidates to fulfill this function. The other possibility is that the C-terminus of SA2 is required for the interaction between Rad21 NT and SA2. The latter is ruled out because the C-terminus truncated SA2 has similar efficiency in coimmunoprecipitating Rad21.

The Rad21 MP but not the Rad21 CT interacting with SA1 and SA2 appears to be contradictory to the findings in yeast, where Scc1/Mcdl NT Separase-cleavage fragment cannot copurify Scc3, whereas the Sccl/Mcdl CT Separase-cleavage fragment can [5]. Both the yeast Sccl/Mcdl (566 aa) and the human $\operatorname{Rad} 21$ (631 aa) have two Separase cleavage sites. The cleavage sites of the yeast Sccl/Mcdl are at $\mathrm{R}^{180}$ and $\mathrm{R}^{268}$, respectively $[31,32]$. The sizes of the Separase-cleavage fragments of Scc1/Mcdl NT (1-180 aa) and $\operatorname{Rad21~NT~(1-172~aa)~are~}$ similar, but the Separase-cleavage fragments of yeast Sccl/Mcdl CT (269-566 aa) and human $\operatorname{Rad} 21$ CT (450-631 aa) are remarkably different. Although Rad21 CT (451-631 aa) cannot immunoprecipitate $\mathrm{SA} 1 / 2$, its $\mathrm{N}$-terminal extended version of $\operatorname{Rad} 21$ (254-631 aa) can (data not shown). It is possible that the co-purification of yeast Scc3 and Sccl/Mcdl CT is dependent of its long MP, just like the Rad21 MP.

\section{Mutations of SA-binding motifs on Rad21 lead to defect of sister chromatid cohesion}

According to the handcuff model, Smc1, Smc3, and Rad21 form a ring, and two of such rings are dimerized via the Rad21 molecules bridged by SA1/SA2 and potentially other cohesinassociated proteins $[17,18]$. Therefore, $\operatorname{Rad} 21-\operatorname{Rad} 21$ interaction with mutations on MP SA-binding motif appears contradictory to the previous observation that knockdown of SAl/2 reduced the Rad21 dimerization [17]. However, when both the NT and MP of SA-binding motifs were mutated, the interaction between mutant $\operatorname{Rad} 21$ proteins was considerably reduced, which is consistent with our previous studies that suggest SAl/2 functions in bridging the two cohesin rings [17]. We also suggested earlier [17,18] that, besides $\mathrm{SA} 1 / 2$, other cohesin-associated proteins may be involved in the bridging of two cohesin rings. Pds5 A and Pds5B are the most likely candidates because they interact with SAl/2 [33,34]. Pds5A/B can be co-immunoprecipitated by Rad21, which is not affected by the mutation or deletion of MP SA-binding motif (Figure 5A, 6E). However, Rad21-Pds5 interaction is abolished by the mutation of the NT SA-binding motif (Figure 6E), suggesting a tripartite interaction among $\operatorname{Rad} 21, \mathrm{SA} 1 / 2$, and Pds5A/B. Whether SAl/2 directly interacts with the NT SA-binding motif of $\operatorname{Rad} 21$ or requires additional interactions with $\mathrm{Pds} 5 \mathrm{~A} / \mathrm{B}$ remains to be determined. Sororin is another potential candidate, which needs to be further investigated.

Depletion of endogenous Rad21 causes premature chromosomal separation, which can be rescued by ectopic expression of WT Rad21, but not significantly by Rad21 with mutations on both the SA-binding motifs. It is possible that the mutations on SA-binding motifs of Rad21 proteins hinder the two cohesin rings to form handcuff configuration, resulting in the inability to establish or maintain sister chromatid cohesion. How SA1/2 and cohesin-associated proteins cooperatively participate in linking the two cohesin rings would be of interest for future investigation.

\section{Materials and Methods}

\section{Antibodies}

Antibodies used in this study are listed below. Mouse monoclonal antibodies: Flag (Sigma-Aldrich, St. Louis, MO), Myc (Calbiochem, San Diego, CA.), His (BD Biosciences, San Jose, CA), Rad21 [35], SA2 mAb (Santa Cruz Biotech, Santa Cruz, CA), and Actin (Sigma-Aldrich). Rabbit polyclonal antibodies: Flag (Sigma-Aldrich), Myc (Sigma-Aldrich), Smc3 (Bethyl Laboratories, Inc., Montgomery, TX), and Rad21 pAb [35]. Goat polyclonal antibodies: SA1 and SA2 (Novus Biologicals, Littleton, CO), and Smcl $\alpha$ (Santa Cruz Biotech). All secondary antibodies were from Rockland Immunochemicals (Gilbertsville, PA). 


\section{Cell culture}

Sf21 cells (Invitrogen, Carlsbad, CA) were grown in Grace's media (Sigma-Aldrich) supplemented with $10 \% \mathrm{FBS}$ at $27^{\circ} \mathrm{C}$ on a shaker at $110 \mathrm{rpm}$. $293 \mathrm{~T}$ cells (ATCG, Manassas, VA) were grown in DMEM supplemented with $10 \% \mathrm{FBS}$ in a $37^{\circ} \mathrm{C}$ incubator under $95 \%$ humidity and $5 \% \mathrm{CO}_{2}$.

\section{Cloning of SA2 and Rad21 deletion mutants in baculovirus system}

SA2 or Rad21 cDNA was cloned into the pFastBacl vector (Invitrogen). Recombinant viruses were generated according to the Bac-to-Bac Baculovirus Expression System protocol (version D) (Invitrogen).

\section{Cloning of SA2 and Rad21 deletion mutants in mammalian cells}

SA2 (1-1051 aa) was cloned into the pCS2MT vector, and Rad21 deletion mutants were cloned into pFlag CMV2, pCS2MT, or pCruz HA vectors.

\section{Transfection}

Plasmids were transfected into $293 \mathrm{~T}$ cells using the calcium phosphate method [36]. The medium was changed $16 \mathrm{~h}$ after transfection.

\section{In-gel digestion and Mass Spectrometry (MS)}

The SA2 sample was electrophoresed on a SDS-PAGE gel and stained with Coomassie staining solution (Bio-Rad, Berkeley, CA). The band containing the SA2 degradation product was cut out and sent to the Tufts University Core Facility for in-gel digestion and MS analysis.

\section{Co-purification of baculovirus expressed proteins}

Ten million Sf21 cells were harvested $48 \mathrm{~h}$ post-infection and lysed in $1 \mathrm{ml}$ lysis buffer (50 mM Tris-HCl pH 7.4, $50 \mathrm{mM} \mathrm{NaCl}$, $1 \%$ Triton X-100, and $1 \mathrm{mM}$ PMSF). For Ni-NTA co-purification, cell extracts were supplemented to contain $20 \mathrm{mM}$ imidazole and $300 \mathrm{mM} \mathrm{NaCl}$ before being incubated with $30 \mu \mathrm{l} \mathrm{Ni}-\mathrm{NTA}$ resin (Qiagen, Valencia, CA) at $4^{\circ} \mathrm{C}$ for $1 \mathrm{~h}$. The resin was washed three times with $1 \mathrm{ml} \mathrm{Ni-NTA}$ buffer A (50 mM Tris-HCl pH 7.5, $300 \mathrm{mM} \mathrm{NaCl}$, and $20 \mathrm{mM}$ imidazole), and the tagged protein was eluted with $2 \times$ SDS sample buffer $(125 \mathrm{mM}$ Tris-HCl, pH 6.8, 20\% glycerol, 4\% SDS, $0.1 \%$ bromophenol blue, and $100 \mathrm{mM}$ DTT). For Flag beads co-purification, the cell extracts was adjusted to contain $150 \mathrm{mM} \mathrm{NaCl}$ and incubated with $20 \mu \mathrm{l}$ Flag beads at $4^{\circ} \mathrm{C}$ for $3 \mathrm{~h}$. The beads were then washed three times with $1 \mathrm{ml}$ PBS buffer $(137 \mathrm{mM} \mathrm{NaCl}, 2.7 \mathrm{mM} \mathrm{KCl}$, $8.1 \mathrm{mM}$ Na2HPO4, and $1.5 \mathrm{mM} \mathrm{KH} 2 \mathrm{PO} 4, \mathrm{pH} 7.4$ ), and the tagged protein was eluted with $2 \times$ SDS sample buffer.

\section{Purification of the SA2:Rad21 complex}

Two liters of Sf21 cells were co-infected with baculovirus overexpressing the 6xHis tagged SA2 (1-1051 aa) and Flag tagged Rad21 (171-450 aa) at a multiplicity of infection of 4:4. Sf21 cells were harvested $60 \mathrm{~h}$ post-infection. Cell pellets were then suspended in Ni-NTA buffer A (50 mM Tris-HCl pH 7.5, $300 \mathrm{mM} \mathrm{NaCl}$, and $20 \mathrm{mM}$ imidazole). Following lysis and centrifugation, the supernatant was loaded onto Ni-NTA beads (Qiagen). The mixture was gently stirred at $4^{\circ} \mathrm{C}$ for $1 \mathrm{~h}$, followed by an $8 \mathrm{~min}$ centrifugation at 2,000 $\mathrm{xg}$ to separate the beads from the flow through. The beads were then washed by a washing buffer containing $20 \mathrm{mM}$ imidazole. After washing, the complex was eluted by an elution buffer containing $250 \mathrm{mM}$ imidazole.
The eluted protein solution containing the complex was then diluted three times with FPLC buffer A (50 mM Tris-HCl pH 7.5, $1 \mathrm{mM}$ EDTA, $1 \mathrm{mM} \mathrm{NaN}_{3}$, and $10 \%$ glycerol) and loaded onto a $5 \mathrm{ml}$ Hitrap Q column (GE Healthcare, Pittsburgh, PA), which is an anion exchange column. Samples were eluted using an increasing gradient of salt at $\mathrm{pH}$ 7.5. Fractions containing the complex were collected, concentrated, and loaded onto a $24 \mathrm{ml}$ Superose 6 gel filtration column (GE Healthcare). A gel filtration buffer (25 mM Tris-HCl pH 7.5, $200 \mathrm{mM} \mathrm{NaCl,}$ and 5\% glycerol) was used to elute the protein.

\section{Immunoprecipitation and Western blotting}

Protein isolation and immunoprecipitation were performed as reported previously [17]. Cell lysates or IP samples were electrophoresed on 5\%-20\% gradient SDS gels and transferred to nitrocellulose membranes (Bio-Rad). The membranes were blotted with blocking buffer (LI-COR Biosciences, Lincoln, NE) for $1 \mathrm{~h}$ and probed with primary antibody for $1 \mathrm{~h}$, after which the membranes were washed three times with TBST buffer $(20 \mathrm{mM}$ Tris-HCl pH 7.4, $150 \mathrm{~m} \mathrm{NaCl}$, and $0.1 \%$ Tween 20). The membranes were then probed with appropriate secondary antibodies labeled with IRDye 800 or Cy 5.5 for $1 \mathrm{~h}$, followed by three washes with TBST buffer. The membranes were then visualized by an Odyssey infrared scanner (LI-COR Biosciences).

\section{Preparation of chromatin fraction from 293 T cells}

$293 \mathrm{~T}$ cells were lysed, and pellets were collected using centrifugation. The pellets were then suspended in cell lysis buffer containing $1 \mathrm{mM}$ MNase. After sonication, the samples were incubated in $37^{\circ} \mathrm{C}$ water bath for $10 \mathrm{~min}$. The supernatants were collected as the chromatin fraction after centrifugation at $20000 \mathrm{xg}$ for $15 \mathrm{~min}$.

\section{Analytical ultracentrifugation and data analysis}

Sedimentation velocity experiments were performed using a Beckman Coulter Optima XL-A analytical ultracentrifuge. The optical density $(\mathrm{OD})$ values of the samples were obtained against a water reference at $230 \mathrm{~nm}$. The sedimentation velocity runs were performed at 30,000rpm (AN60 Rotor) at $4^{\circ} \mathrm{C}$ for $10 \mathrm{~h}$. One hundred scans were recorded for each sample at a radial step size of $30 \mu \mathrm{m}$. Data analysis was performed with UltraScan version 9.9 (Ultrascan [37], http: //www.ultrascan.uthscsa.edu). Time invariant noise and radially invariant noise were subtracted from the sedimentation velocity data by 2-dimentional spectrum analysis [38]. G(s) sedimentation coefficient distributions were obtained with the enhanced van Holde-Weischet analysis [39].

\section{SiRNA and transfection}

Silencer Negative Control siRNA (Applied Biosystems, Foster City, CA) was used as a negative control. Rad21 siRNA (Qiagen) was transfected into cells using DharmaFECT ${ }^{\mathrm{TM}} 1$ (Dharmacon, Lafayette, CO).

\section{Metaphase chromosome spread}

Cells were treated by trypsin, after which they were centrifuged at $800 \mathrm{~g}$ for $6 \mathrm{~min} .10 \mathrm{ml}$ of a pre-warmed hypotonic solution $(0.075 \mathrm{M} \mathrm{KCl})$ was added to gently agitate the pellets, followed by incubation at $37^{\circ} \mathrm{C}$ for 15 min to break the membrane. One $\mathrm{ml}$ of fresh fixative (methanol: glacier acetic acid $=3: 1$ ) was then added before the cells were centrifuged at $800 \mathrm{~g}$ for $6 \mathrm{~min}$ and the supernatants were discarded. Four $\mathrm{ml}$ of fixative was added to each sample before they were incubated at room temperature for $30 \mathrm{~min}$. Fixative was changed twice and cells were re-suspended in 
$200 \mu$ fixative after the final centrifugation. To create slides, $30 \mu \mathrm{l}$ mixture was taken from each sample and dropped on angled slides from 10 inches above. The slides were then air-dried and stained in Giemsa solution for 10 min before being rinsed with water ten times. About 100 metaphase cells were counted for each treatment using a Zeiss AxioSkop 40 microscope (Carl Zeiss, Thornwood, NY).

\section{Data analysis}

All the experiments described in this study were performed multiple times ( 2 or more times) and the representative figures are shown. The results of premature separation of chromatids were analyzed using Student's $t$-test.

\section{Supporting Information}

Figure S1 Identification of the SA2 degradation product. (A) Western blot of purified SA2 full length protein. The 6xHis tag at the $\mathrm{N}$-terminal of full length SA2 was detected by $6 \mathrm{xH}$ is $\mathrm{mAb}$. There is a $\sim 120 \mathrm{kDa}$ degradation product also containing the N-terminal tag. (B) In-gel digestion and peptide identification by HPLC/MS. T ${ }^{122}$ is the last amino acid identified by MS. The peptide coverage at the Nterminal region is not shown. The lines below the amino acid sequence indicate the peptides were identified by mass spectrometry. (TIF)

Figure S2 Secondary structure prediction for Rad21 (171450 aa). $\operatorname{Rad} 21$ amino acid sequence is colored based on residue types (e.g. blue for positively charged, red for negatively charged, green for hydrophobic, silver for polar, etc). PROF_sec predicts the secondary structure $(\mathrm{H}=$ Helix). Rel_Sec shows the reliability index of the PROF_sec prediction $(0=$ low, $9=$ high $)$.

(TIF)

Figure S3 Mutations on middle part SA1/2-binding motif of Rad21 do not affect Rad21-Rad21 interact and co-immunoprecipitate Smcl/3. $293 \mathrm{~T}$ cells were transfected with pCM2 MT $\operatorname{Rad} 21$ (and pFlag GMV2 $\operatorname{Rad} 21$ for $(\mathrm{B})$ ). Empty vector was used as control. Co-immunoprecipitation was performed using whole cell lysate. (A) Immunoblotting shows the cohesin core subunits including endogenous Rad21 were co- immunoprecipitated by Myc-Rad21 WT and mutants. (B) Immunoblotting of the co-IP of Flag-Rad21 and Myc-Rad21, which does not affect by the mutation on the middle part of SAl/2 binding motif of Rad21.

(TIF)

\section{References}

1. Hagstrom KA, Meyer BJ (2003) Condensin and cohesin: more than chromosome compactor and glue. Nat Rev Genet 4: 520-534.

2. Losada A, Yokochi T, Kobayashi R, Hirano T (2000) Identification and characterization of $\mathrm{SA} / \mathrm{Scc} 3 \mathrm{p}$ subunits in the Xenopus and human cohesin complexes. J Cell Biol 150: 405-416.

3. Uhlmann F (2001) Chromosome cohesion and segregation in mitosis and meiosis. Curr Opin Cell Biol 13: 754-761.

4. Anderson DE, Losada A, Erickson HP, Hirano T (2002) Condensin and cohesin display different arm conformations with characteristic hinge angles. J Cell Biol 156: 419-424.

5. Haering CH, Lowe J, Hochwagen A, Nasmyth K (2002) Molecular architecture of SMC proteins and the yeast cohesin complex. Mol Cell 9: 773-788.

6. Lowe J, Cordell SC, van den EF (2001) Crystal structure of the SMC head domain: an ABC ATPase with 900 residues antiparallel coiled-coil inserted. J Mol Biol 306: 25-35.

7. Schmitz J, Watrin E, Lenart P, Mechtler K, Peters JM (2007) Sororin is required for stable binding of cohesin to chromatin and for sister chromatid cohesion in interphase. Curr Biol 17: 630-636.

8. Rankin S, Ayad NG, Kirschner MW (2005) Sororin, a substrate of the anaphasepromoting complex, is required for sister chromatid cohesion in vertebrates. Mol Cell 18: 185-200.
Figure S4 Immunoblotting shows co-immunoprecipitation of Myc-SA1 and Flag-Rad21 WT and mutants. $293 \mathrm{~T}$ cells were transfected with pCS2 MT SA1 and pFlag CMV2 Rad21 WT or mutant with mutations on middle part of SAl/2-binding motif. Co-immunoprecipitation was performed using whole cell lysate. EV: empty vector; WT: wild type; SM: L385A; DM: L385A T390A; TM: L385A F389A T390A; Del: del(383-392 aa). (TIF)

Figure S5 N-terminal Rad21 (1-172 aa) and middle part of Rad21 (173-450 aa) contains SAl/2-binding motif. 293 T cells were transfected with the appropriate plasmids as shown. IP was performed using cell lysates $40 \mathrm{~h}$ after transfection. (A) Schematic illustration shows the Rad21 truncated mutants. The Separase cleavage sites at 172 and 450 (arrows) and SAl/2-binding motif at 383-392 aa (rectangle block) are shown. WT: wild type; NT: Nterminus; MP: middle part; CT: C-terminus. (B) Rad21 NT coimmunoprecipitates itself as well as SA1, SA2 and endogenous Rad21 (lane 5), but not Smc1 and Smc3. (G) Flag- and HA-Rad21 MP co-immunoprecipitate each other as well as SA1 and SA2, but fail to co-immunoprecipitate Smc1, Smc3 and Rad21 (lane 4). (D) Flag- and HA-Rad21 CT co-immunoprecipitate Smcl and Smc3, but fail to co-IP each other and SAl/2 (lane 4).

(TIF)

Figure S6 Helical wheel illustration of the $\operatorname{Rad} 21$ 383-392 aa. $\mathrm{L}^{385}$ and $\mathrm{F}^{389}$ are next to each other in the helix. The helical wheel was created using the following website: http://rzlab.ucr.edu/ scripts/wheel/wheel.

cgi? sequence $=$ ABCDEFGHIJLKMNOP\&submit $=$ Submit $\quad(\mathrm{Zi}-$ dovetzki, R., Rost, B., Armstrong, D. L., and Pecht, I. (2003) Biophys. Chem. 100, 555-575).

(TIF)

\section{Acknowledgments}

We thank Dr. B. Lee Ligon for editing of the manuscript.

\section{Author Contributions}

Performed the experiments: YJ NZ QM. Analyzed the data: NZ YJ BD YJT DP. Contributed reagents/materials/analysis tools: BD. Wrote the paper: NZ YJ. Conceived the project: DP. Designed the experiments: YJ NZ YJT DP. Contributed to the manuscript: BD YJT DP.
9. Panizza S, Tanaka T, Hochwagen A, Eisenhaber F, Nasmyth K (2000) Pds5 cooperates with cohesin in maintaining sister chromatid cohesion. Curr Biol 10: $1557-1564$.

10. Nishiyama T, Ladurner R, Schmitz J, Kreidl E, Schleiffer A, et al. (2010) Sororin mediates sister chromatid cohesion by antagonizing Wapl. Cell 143: 737-749.

11. Losada A, Yokochi T, Hirano T (2005) Functional contribution of Pds5 to cohesin-mediated cohesion in human cells and Xenopus egg extracts. J Cell Sci 118: 2133-2141.

12. Zhang N, Panigrahi AK, Mao Q, Pati D (2011) Interaction of Sororin with pololike kinase 1 mediates the resolution of chromosomal arm cohesion. J Biol Chem 286: 41826-41837.

13. Zhang N, Pati D (2012) Sororin is a master regulator of sister chromatid cohesion and separation. Cell Cycle 11: 2073-2083.

14. Panigrahi AK, Zhang N, Mao Q, Pati D (2011) Calpain-1 cleaves rad21 to promote sister chromatid separation. Mol Cell Biol 31: 4335-4347.

15. Haering CH, Nasmyth K (2003) Building and breaking bridges between sister chromatids. Bioessays 25: 1178-1191.

16. Hauf S, Waizenegger IC, Peters JM (2001) Cohesin cleavage by separase required for anaphase and cytokinesis in human cells. Science 293: 1320-1323.

17. Zhang N, Kuznetsov SG, Sharan SK, Li K, Rao PH, et al. (2008) A handcuff model for the cohesin complex. J Cell Biol 183: 1019-1031. 
18. Zhang N, Pati D (2009) Handcuff for sisters: a new model for sister chromatid cohesion. Cell Cycle 8: 399-402.

19. Panigrahi AK, Pati D (2009) Road to the crossroads of life and death: Linking sister chromatid cohesion and separation to aneuploidy, apoptosis and cancer. Crit Rev Oncol Hematol.

20. Solomon DA, Kim T, az-Martinez LA, Fair J, Elkahloun AG, et al. (2011) Mutational inactivation of STAG2 causes aneuploidy in human cancer. Science 333: 1039-1043

21. Canudas S, Smith S (2009) Differential regulation of telomere and centromere cohesion by the Scc3 homologues SA1 and SA2, respectively, in human cells. J Cell Biol 187: 165-173.

22. Shintomi K, Hirano T (2009) Releasing cohesin from chromosome arms in early mitosis: opposing actions of Wapl-Pds5 and Sgol. Genes Dev 23: 2224-2236.

23. Rost B, Yachdav G, Liu J (2004) The PredictProtein server. Nucleic Acids Res 32: W321-W326.

24. Linding R, Russell RB, Neduva V, Gibson TJ (2003) GlobPlot: Exploring protein sequences for globularity and disorder. Nucleic Acids Res 31: 37013708.

25. Arumugam P, Gruber S, Tanaka K, Haering CH, Mechtler K, et al. (2003) ATP hydrolysis is required for cohesin's association with chromosomes. Curr Biol 13: 1941-1953.

26. Gimenez-Abian JF, Sumara I, Hirota T, Hauf S, Gerlich D, et al. (2004) Regulation of sister chromatid cohesion between chromosome arms. Curr Biol 14: 1187-1193.

27. Sumara I, Vorlaufer E, Gieffers C, Peters BH, Peters JM (2000) Characterization of vertebrate cohesin complexes and their regulation in prophase. J Cell Biol 151: 749-762.

28. Sumara I, Vorlaufer E, Stukenberg PT, Kelm O, Redemann N, et al. (2002) The dissociation of cohesin from chromosomes in prophase is regulated by Pololike kinase. Mol Cell 9: 515-525.
29. Hauf S, Roitinger E, Koch B, Dittrich CM, Mechtler K, et al. (2005) Dissociation of Cohesin from Chromosome Arms and Loss of Arm Cohesion during Early Mitosis Depends on Phosphorylation of SA2. PLoS Biol 3: e69.

30. Pezzi N, Prieto I, Kremer L, Perez Jurado LA, Valero C, et al. (2000) STAG3, a novel gene encoding a protein involved in meiotic chromosome pairing and location of STAG3-related genes flanking the Williams-Beuren syndrome deletion. FASEB J 14: 581-592.

31. Uhlmann F, Lottspeich F, Nasmyth K (1999) Sister-chromatid separation at anaphase onset is promoted by cleavage of the cohesin subunit Sccl. Nature 400: $37-42$.

32. Uhlmann F, Wernic D, Poupart MA, Koonin EV, Nasmyth K (2000) Cleavage of cohesin by the CD clan protease separin triggers anaphase in yeast. Cell 103: 375-386.

33. Kueng S, Hegemann B, Peters BH, Lipp JJ, Schleiffer A, et al. (2006) Wapl controls the dynamic association of cohesin with chromatin. Cell 127: 955-967.

34. Rowland BD, Roig MB, Nishino T, Kurze A, Uluocak P, et al. (2009) Building sister chromatid cohesion: smc3 acetylation counteracts an antiestablishment activity. Mol Cell 33: 763-774.

35. Pati D, Zhang N, Plon SE (2002) Linking sister chromatid cohesion and apoptosis: role of Rad21. Mol Cell Biol 22: 8267-8277.

36. Freshney RI (2000) Culture of animal cells: a manual of basic technique. New York:Wiley-Liss, Inc.

37. Demeler B (2005) Ultrascan: a comprehensive data analysis software package for analytical ultracentrifugation experiments. In: Scott DJ, Harding SE, Rowe Aj, editors. In Modern Ananlytical Ultracentrifugation: Techniques and Methods. London: Royal Society of Chemistry, pp. 210-229.

38. Brookes E, Cao W, Demeler B (2010) A two-dimensional spectrum analysis for sedimentation velocity experiments of mixtures with heterogeneity in molecular weight and shape. Eur Biophys J 39: 405-414.

39. Demeler B, van Holde KE (2004) Sedimentation velocity analysis of highly heterogeneous systems. Anal Biochem 335: 279-288. 